\title{
Editorial
}

\section{Cooperation between neuropsychology researchers and ethical committees: Room for improvement?}

\author{
Roberto Cubelli ${ }^{a}$ and Sergio Della Sala ${ }^{b, *}$ \\ ${ }^{a}$ Department of Psychology and Cognitive Sciences, University of Trento, Italy \\ ${ }^{\mathrm{b}}$ Human Cognitive Neuroscience, Psychology, University of Edinburgh, UK
}

In this issue we launch a debate on the functioning of ethical committees (see Discussion Forum, this issue, following Baron's 2015 target article). We need ethics, we value ethical principles as expressed by the 1948 Universal Declaration of Human Rights; hence, we support ethical committees (ECs). Yet, more often than desirable, we consider the requests by our local ethical committee as an extra hurdle to go through to carry out our research. This is dangerous, as the discernment on ethical principles that we accomplished in the last 50 years could be hampered. This sentiment is unfortunately reinforced by the awkward procedures enacted by some ECs. We have once been refused permission to carry out a study as we compiled our request on a pink rather than yellow printed form. A colleague was summoned to explain why they used Times New Roman font for their information sheet rather than the apparently much clearer Helvetica. Capricious and groundless requests such as these mar the dependable cooperation between researchers and members of ECs. However, they are no reason to dismiss the principles underlining the role of such committees.

Bewildering requests by EC should not become the pretext to overlook established ethical principles, or for pardoning researchers' ignorance of such principles.

The updated version of the Declaration of Helsinki (Fortaleza, Brazil, 2013) states:

"The research protocol must be submitted for consideration, comment, guidance and approval to the concerned research ethics committee before the study begins. This committee must be transparent in its functioning, must be independent of the researcher, the sponsor and any other undue influence and must be duly qualified. It must take into consideration the laws and regulations of the country or countries in which the research is to be performed as well as applicable international norms and standards but these must not be allowed to reduce or eliminate any of the protections for research subjects set forth in this Declaration. The committee must have the right to monitor ongoing studies. The researcher must provide monitoring information to the committee, especially information about any serious adverse events. No amendment to the protocol may be made without consideration and approval by the committee. After the end of the study, the researchers must submit a final report to the committee containing a summary of the study's findings and conclusions." (Article 23, our underlining).

The Oviedo Convention on Human Rights and Biomedicine (1997) advises that a study on humans could be undertaken only after several conditions are met, among which the following criterion:

The research project has been approved by the competent body after independent examination of its scientific merit, including assessment of the importance of the aim of the research, and multidisciplinary review of its ethical acceptability (Article 16).

The UK research funding council ESRC states that

"Research ethics are about incorporating ethical principles into research. They may involve a balance between and within principles and practices and at all stages, includes all those involved from inception of research through to completion and publications of research and beyond."

The task of ECs is to make sure that these principles are implemented. They should ensure on one hand the accretion of scientific knowledge via new empirical evidence, on the other the safeguard of psycho-physical wellbeing of the participating individuals.

\footnotetext{
* Corresponding author.

E-mail address: sergio@ed.ac.uk (S. Della Sala). http://dx.doi.org/10.1016/j.cortex.2015.04.016

0010-9452/@ 2015 Elsevier Ltd. All rights reserved.
} 
ECs guarantee both the researcher's freedom to investigate and the participant's freedom to decide. To decide, the participants need to be properly and accurately informed about the study by the researcher. The researcher needs access to the participant's data, including relevant personal ones. Should these two reciprocal freedoms conflict, the committee is called to propose solutions mediating between them. The main task of the ECs is therefore to render the study process fully explicit, feasible and to support the researcher to become more aware of the multifarious ethical aspects and potential pitfalls that such process might entail.

ECs must help researchers in bettering their study paradigms. This welcoming approach is often not what researchers encounter. We are sure that examples of malfunctioning of ECs could be the focus of entertaining conversation at most scientific conventions. As many other researchers, we also experienced some excess of rigour. For instance in one occasion, we have been taken to task in a neuroimaging proposal geared at studying memory for words as we intended to recruit participants whose mother tongue was English. Apparently we should have made available online translations from all other languages to avoid discrimination in selecting participants. In other instances such firmness translates in an excess of leniency; the amendment requested by a colleague who had been granted permission to use a $3 \mathrm{~T}$ MRI but wanted instead to use a $7 \mathrm{~T}$ in a single case with the same protocol, was disdained with the argument that it was just another scanner. Both cases reveal some incompetence. The former exemplifies the confusion between the ethical obligation to avoid inequality and the methodological requirements of inclusion and exclusion criteria. The latter shows the lack of understanding of the difference of the potential risks linked with the two magnetic fields.

The above anecdotes exemplify the difficulty in communication between human neuroscience researchers and (some) ECs. Part of this difficulty springs from the lack of specific paths to vet ethical issues connected with human neuroscience research projects. In most countries animal research or clinical trials are precisely regulated, hence ECs accrue competence and standardize their reaction in scrutinizing projects in these areas. Human neuroscience projects do not fall in either of these two established categories. ECs are therefore less equipped or even less available to inspect them. This normative vacuum becomes particularly serious in the case of neuropsychological research projects involving patients. The disparity in the handling of applied and basic research is causing the progressive curtailing of neuropsychological research within hospital sites and of the potentially fruitful collaboration between academics and clinicians (Della Sala, Cubelli, \& McIntosh, 2015). Neuropsychological research in clinical settings elicit specific ethical issues which deserve deliberations, discussions and possibly solutions by both the relevant scientific communities and the various ECs. For example, most neuropsychological studies carry little or no benefit for the individual participants, thus soliciting the debate on direct incentives and freedom to volunteering.
The idiosyncrasies of human neuroscience research exacerbate some inherent problems to the functioning of ECs. We will mention three of these problems. First, the progressive bureaucratization which leads to inflexible procedures which do not consider the specificity of different research areas; unyielding forms requesting irrelevant, and sometimes misleading information; lack of definite deadlines causing long delays, uncertainties and frustration; scant justification of decisions taken and in supporting requests for further amendments, preventing a constructive rebuttal. This phenomenon is also due to the widespread compliance with a defensive attitude aimed at protecting the institutions against study participants as potential plaintiffs. Second, the expertise of members of ECs and their selection: rarely do ECs include a member with some knowledge of the specific methodologies used in human neuroscience. Consider the complexity of examining the application for a single case study, which entails unusual statistical approaches, is based on a wide range of tests (most of which cannot be a priori identified), and requires a very swift response. The third problem concerns the symmetrical competence of researchers, who too often know little about the ethical issues embedded in their own research. These issues are often not straightforward and could be counterintuitive. We could do much better in exposing human neuroscience and neuropsychology students to a formal training in ethical issues within their education.

Ideally, ECs and researchers should collaborate towards improving research designs, breaking away from the relative prejudices, whereby researchers conceiving ECs as yet another illegitimate annoyance to be dealt with, and ECs viewing researchers as potential tricksters solely geared at satisfying their own egocentric goals.

The discussion which ensues is twofold: the reappraisal of the fundamental tenets of research ethics and the way ECs enact the principles deriving from these tenets. Since WWII the deontological approach has dominated the discussion on research ethics, recently a more utilitarian view has emerged. These two approaches address differently the various ethical issues inherent to research, including neuropsychological research, from informed consent to confidentiality. The second arm of the proposed discussion revolves around how ECs should operate to meet at the same times the needs of neuropsychological researchers and those of the participants to their studies.

We hope that this Discussion Forum will help a bit to overcome the current hiatus between research groups and their relevant ECs.

\section{R E F E R E N C E S}

Baron, J. (2015). Some fallacies of human-subjects protection, and some solutions. Cortex, 65, 246-254.

Della Sala, S., Cubelli, R., \& McIntosh, R. D. (2015). Clinical postcards. Cortex, 64, A1-A3. 